\title{
High-throughput Sequencing Analysis and Function Prediction of Lung Microbiota in Healthy Rats
}

\author{
Xiaoyu $\mathbf{L i}^{*}$ \\ Laboratory of Innovation, Basic Medical Experimental Teaching Center, Chongqing Medical University, Chongqing, China
}

Zhibang Yang

Laboratory of Pathogenic Biology and Immunology, School of Basic Medicine, Chongqing Medical University, Chongqing, China

Jia Liu

Basic Medical Experimental Teaching Center, Chongqing Medical University, Chongqing, China

\author{
Ying Jiang
}

Laboratory of Pathogenic Biology and Immunology, School of Basic Medicine, Chongqing Medical University, Chongqing, China

\begin{abstract}
To form the basis of a respiratory disease model in rats by investigating the microbial distribution and composition in the lower respiratory tracts of normal rats. Methods: DNA was extracted from the intestine, trachea, bronchus and lung samples collected from healthy rats under sterile conditions. The 16S rDNA V4-V5 region was sequenced using Illumina high-throughput technology. Results: The sequencing results showed that there was no significant difference in abundance and species diversity of microbiota between the lower respiratory and the intestine. The microbiota structure analysis showed samples from lungs and intestinal shared similarity. However, the dominant species at the levels of phylum, family, and genus diverged. The similarity analysis showed that the lung microbiota were different from the intestines. The linear discriminant analysis showed significantly different species in different tissues; function prediction also showed different microbiota function in different tissues. Conclusions: These results suggest that bacterial colonization depends on the sample's anatomical location. The human pathogen Acinetobacter lwoffii was also detected in the rat lower respiratory tract samples.
\end{abstract}

Keywords: Illumina sequencing; Lung microbiota; Lower respiratory tract; Intestinal; 16S rRNA; Rats.

(c) (9) CC BY: Creative Commons Attribution License 4.0

\section{Introduction}

Our understanding of the interaction between host health and human microbiome has made great advances in recent years. This is especially true for the gut microbiota, which has been reported to be closely associated with inflammatory bowel disease, colon cancer, obesity, diabetes, and other diseases [1-4]. In the National Institutes of Health's Human Microbiome Project (HMP), the gut microbiota was the most important and most widely studied part of the human microbiome. However, the respiratory tract was not included in the HMP. The traditional view holds that the healthy lower respiratory tracts, especially the bronchioles and lungs, are free of bacteria. However, the continuing maturation of molecular biology and culture-free microbial detection technologies have revealed a certain number of bacteria colonizing the lungs; these bacteria are known as lung microbiota [5]. In recent studies, significant differences in lung microbiota have been found between diseased and healthy conditions. This suggests that lung microbiota, much like the gut microbiota, is involved in the development of diseases such as asthma, pulmonary cystic fibrosis, lung cancer and other diseases [6-9].

With the increasing demand of medical research, animal models are widely used to study the mechanisms of human disease and development, drug screens and treatment evaluations. In resent researches the existence of microbes in the lungs of experimental dogs and sheep have been reported and the microbial community structure also been analyzed [10, 11]. However, despite being the most widely used animal model, the composition and distribution of lung microbiota in the rat has not been reported. To determine if a consistent lung microbiota is present in healthy rats, the trachea, bronchus and lungs of healthy rats were collected under sterile conditions and subjected to DNA extraction methods. DNA was analyzed with Illumina high-throughput sequencing of the $16 \mathrm{~S}$ rDNA V4-V5 region. Intestinal samples were also collected to investigate possible correlations between lung microbiota and gut microbiota.

\section{Materials and Methods}

\subsection{Experimental Animals}

Three female Sprague-Dawley rats without mating, weighing $200 \pm 20 \mathrm{~g}$, were randomly selected from the Experimental Animal Center of Chongqing Medical University [SCXK- (Yu) 2012-0001]. Animals had access to clean food and water ad libitum and were fed under daylight-simulating light for 12 hours followed by 12 hours of 
darkness (room temperature $23 \pm 2{ }^{\circ} \mathrm{C}$, relative humidity of $5070 \%$, natural ventilation). All rats were fed in the laboratory after birth.

All experiments with rats were performed in accordance with the "Laboratory Animal-Requirements of Environment and Housing Facilities" (GB 14925-2001). The experiments were approved by the Animal Laboratory Administrative Center and the Institutional Ethics Committee of Chongqing Medical University (License number: SCXK YU 2012-0001) and adhered to Chongqing Administration Rule of Laboratory Animals and the National Institutes of Health Guidelines.

\subsection{Sample Collection}

Rats were sacrificed and dissected under sterile conditions. The left lung tissue of the rats was labeled group A; the pharynx to the hilum of the tracheal and bronchial tissues were labeled group B. The specimen was washed with sterile saline water which was then absorbed by bacteria-free absorbent paper. A $1 \mathrm{~cm}$ intestinal section was taken as another specimen and labeled as group C. To rule out the effect of foodborne pathogenic bacteria when testing the intestinal bacteria, the intestinal segments were cut longitudinally with sterile scissors to remove the contents of the intestines. Samples were then washed three times with sterile saline and then treated with sterile absorbent paper to remove moisture. All samples were immediately stored in sterile EP tubes at $-80{ }^{\circ} \mathrm{C}$.

\subsection{DNA Extraction and PCR Amplification}

Total microbial DNA was extracted from all samples with the TansStart FastPfu DNA Kit (Transgene Biotech, Beijing China). The V4-V5 region of the bacterial $16 \mathrm{~S}$ ribosomal RNA gene were amplified by PCR $\left(95^{\circ} \mathrm{C}\right.$ for 5 min, followed by 27 cycles at $95{ }^{\circ} \mathrm{C}$ for $30 \mathrm{~s}, 55^{\circ} \mathrm{C}$ for $30 \mathrm{~s}$, and $72{ }^{\circ} \mathrm{C}$ for $\left.45 \mathrm{~s}\right)$ and a final extension $\left(72{ }^{\circ} \mathrm{C}\right.$ for 10 min) with primers 515F 5'-barcode-GTGCCAGCMGCCGCGG-3' and 907R 5'-CCGTCAATTCMTTTRAGTTT-3' (Sangon Biotech, Shanghai China). The barcode is an eight-base sequence unique to each sample. PCR reactions were performed in triplicate $20 \mu \mathrm{L}$ mixtures which contained $4 \mu \mathrm{L}$ of $5 \times$ FastPfu Buffer, $2 \mu \mathrm{L}$ of $2.5 \mathrm{mM}$ dNTPs, $0.8 \mu \mathrm{L}$ of each primer $(5 \mu \mathrm{M}), 0.4 \mu \mathrm{L}$ of FastPfu Polymerase, and $10 \mathrm{ng}$ of template DNA. After electrophoresis in a $2 \%$ agarose gel, PCR product amplicons were extracted with the AxyPrep DNA Gel Extraction Kit (Axygen Biosciences, Union City, CA, U.S.) and purified by Tris-HCL. .

\subsection{S Rdna V4-V5 Illumina High Throughput Sequencing}

The 16S rDNA V4-V5 PCR amplification product was sent to the Shanghai Major Medical Laboratory Ltd. for Illumina high-throughput sequencing. Purified amplicons were pooled at equimolar concentrations and paired-end sequenced $(2 \times 250)$ on an Illumina HiSeq platform according to the standard protocols. Raw fastq files were demultiplexed and quality-filtered using QIIME (version 1.17) with the following criteria. (i) The 250 bp reads were truncated at any site receiving an average quality score $<20$ over a 10-bp sliding window, discarding the truncated reads that were shorter than $50 \mathrm{bp}$. (ii) Exact barcode matching, 2 nucleotide mismatches in primer matching, and reads containing ambiguous characters were removed. (iii) Only sequences that overlap longer than $10 \mathrm{bp}$ were assembled according to their overlap sequence. Reads that could not be assembled were discarded.

\subsection{Microbial Function Prediction}

To further predict the metagenomic functional capacity of the rat's lung microbiota, the OTU table was used to generate the inferred metagenomic data by using PICRUSt (version 1.1.0). The OTU data were normalized by copy number, respectively, and predicted functional pathways were annotated by using the Kyoto Encyclopedia of Genes and Genomes (KEGG). The KEGG orthology and three-level pathway tables were obtained according to the green genes databases and KEGG orthology copy number.

\subsection{Statistical Analysis}

The data were analyzed by $\mathrm{R}$ software ( $\mathrm{R}$ version 3.2.3, The $\mathrm{R}$ Foundation). The difference between the two groups was analyzed by randomized grouping analysis and subjected to Tukey's post hoc test. P values less than 0.05 were regarded as statistically significant.

\section{Results}

\subsection{Alpha-Diversity Analysis}

To investigate the abundance and diversity of lung microbiota in rats, the bacterial DNA sequencing data were analyzed with single sample diversity analysis. The Ace index, Chao index, Shannon index, Simpson index and the Coverage index for each sample are shown in Table 1. The diversity indices were analyzed by a randomized blocking design analysis; the results showed that there were no significant differences in the Ace, Chao and Shannon indices of each group $(\mathrm{pAce}=0.940, \mathrm{pChao}=0.972$, pShannon $=0.815)$. 
Table-1. Species Diversity Analysis Of The Bacterial Community

\begin{tabular}{l|l|l|l|l|l|l|l}
\hline Sample \# & Reads & OTUs & Ace & Chao & Shannon & Simpson & Coverage \\
\hline A1 & 43333 & 676 & 727 & 726 & 4.78 & 0.0221 & 0.998085 \\
\hline A2 & 35329 & 575 & 638 & 641 & 3.89 & 0.0596 & 0.997198 \\
\hline A3 & 44383 & 504 & 575 & 588 & 3.19 & 0.0989 & 0.997702 \\
\hline B1 & 36525 & 660 & 730 & 744 & 4.73 & 0.0241 & 0.997262 \\
\hline B2 & 42932 & 647 & 710 & 728 & 4.26 & 0.0479 & 0.997717 \\
\hline B3 & 38745 & 319 & 429 & 428 & 2.75 & 0.1155 & 0.997264 \\
\hline C1 & 32216 & 541 & 608 & 634 & 3.36 & 0.2173 & 0.997051 \\
\hline C2 & 37313 & 559 & 635 & 651 & 3.68 & 0.119 & 0.997266 \\
\hline C3 & 40703 & 570 & 630 & 654 & 3.82 & 0.1226 & 0.997740 \\
\hline
\end{tabular}

Tukey's post hoc test revealed that there was not a significant difference in the amount of bacteria between lung and intestinal samples ( $p>0.05$ ). This finding suggested there was not a difference in the abundance and diversity of species in the upper respiratory and intestinal tracts. The Coverage index of each sample was more than 0.997, which suggested that the sample coverage of sequencing methods greater than $99.7 \%$ can fully reflect the real situation of bacteria in the sample.

\subsection{Taxonomic Composition}

At the phylum level, 16 phyla were detected from the nine samples. Nine of these 16 phyla, including Firmicutes, Bacteroidetes, Proteobacteria, Spirochaetae, were detected in all samples. Firmicutes was dominant in all samples. The bacteria in the lungs, trachea, and bronchial were dominated by Firmicutes, Bacteroidetes, Proteobacteria, while bacteria in the intestine were dominated by Firmicutes, Bacteroidetes, Spirochaetae. The bacteria detected in each sample at the phylum level are shown in Fig 1.

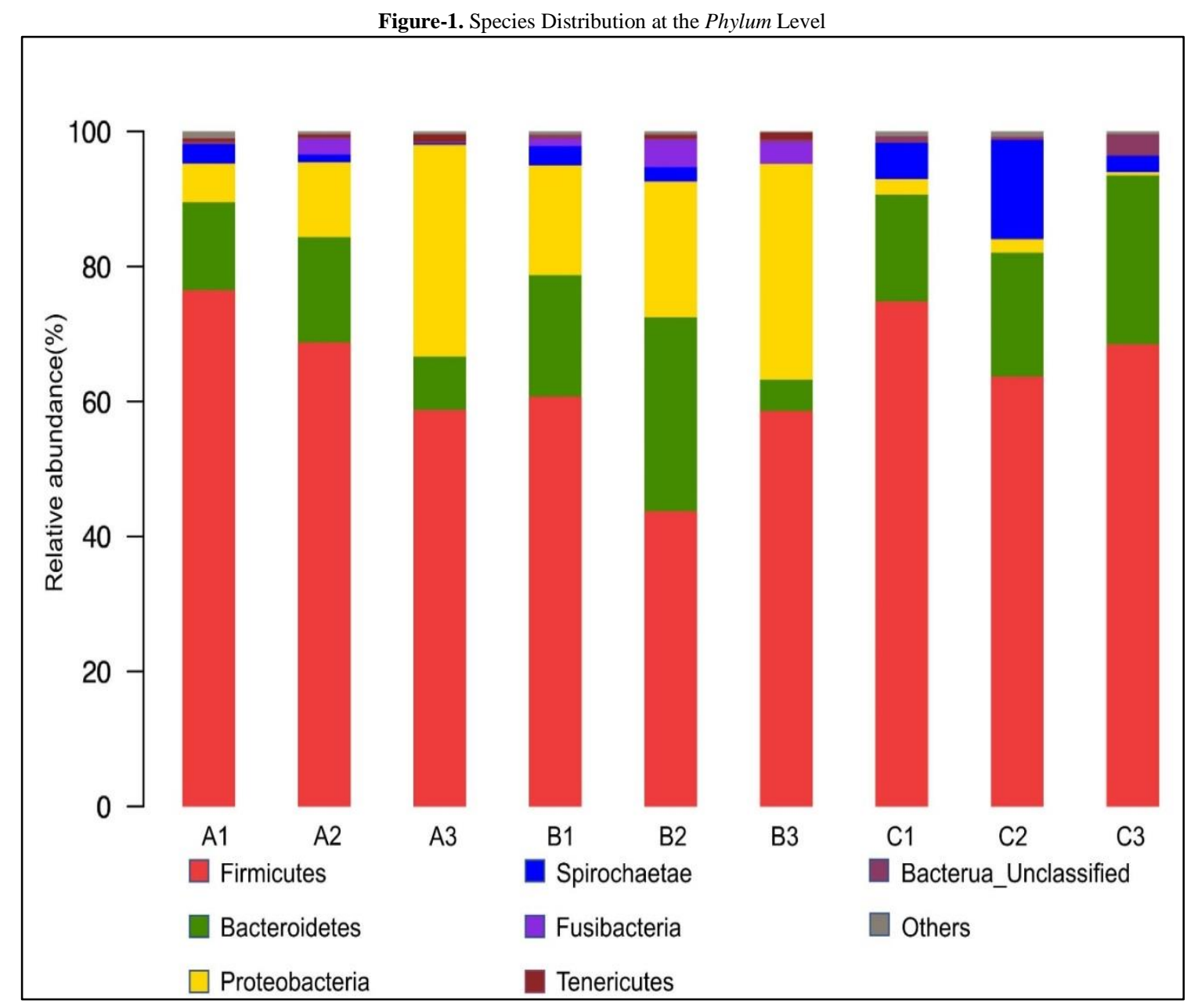

At the family level, a total of 97 families were detected in all samples collectively. The number of families in each individual sample ranged from 53-91. The average number of families detected in the lung samples was 78 . Tracheal and bronchial samples had an average of 76 families, while the intestinal samples had an average of 56. These results indicate that the lower respiratory tract had more bacterial families than the intestinal, suggesting a higher diversity in the colonization of bacteria in lower respiratory tract. The genus Lactobacillus played a leading role in all intestinal samples. The dominant bacterial family and genus in each sample is shown in Figure-2. 
Figure-2. Species Distribution at the Family and Genus Level

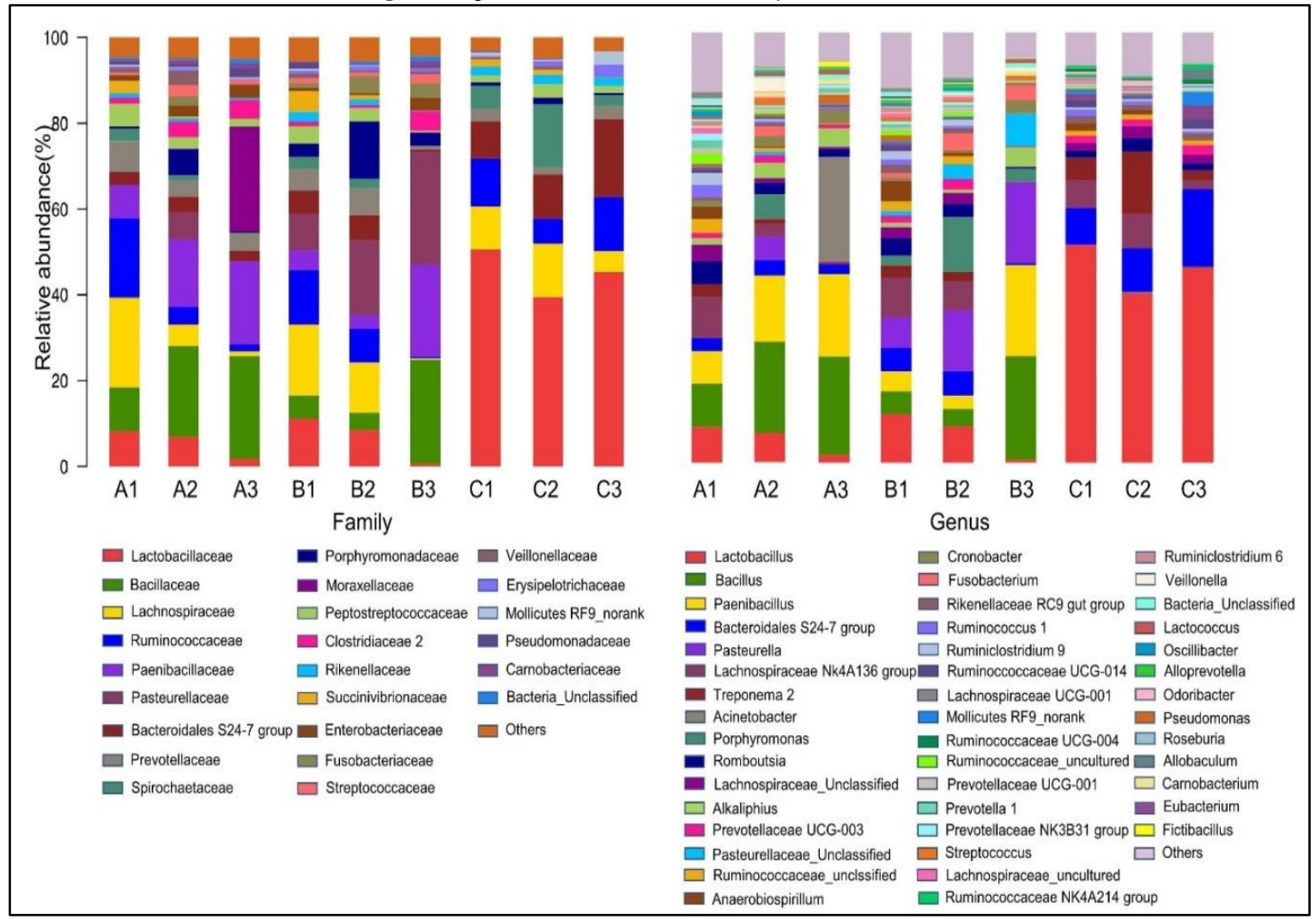

\subsection{Differential Analysis}

To analyze the difference in bacterial communities between samples, linear discriminant analysis effect size (LEfSE) was preformed to obtain the LDA scores of the bacteria. As shown in Fig. 3, the most prominent phyla in group C were Bacteroides, Hymenoscyphus and Rikenellaceae. The most prominent phyla in group B were Proteobacteria, $\gamma$ - Proteobacteria and Caulobacter. The most prominent phyla in group A were Bacillus, Moraxella and Acinetobacter.

Figure-3. The LEfSe LDA scores

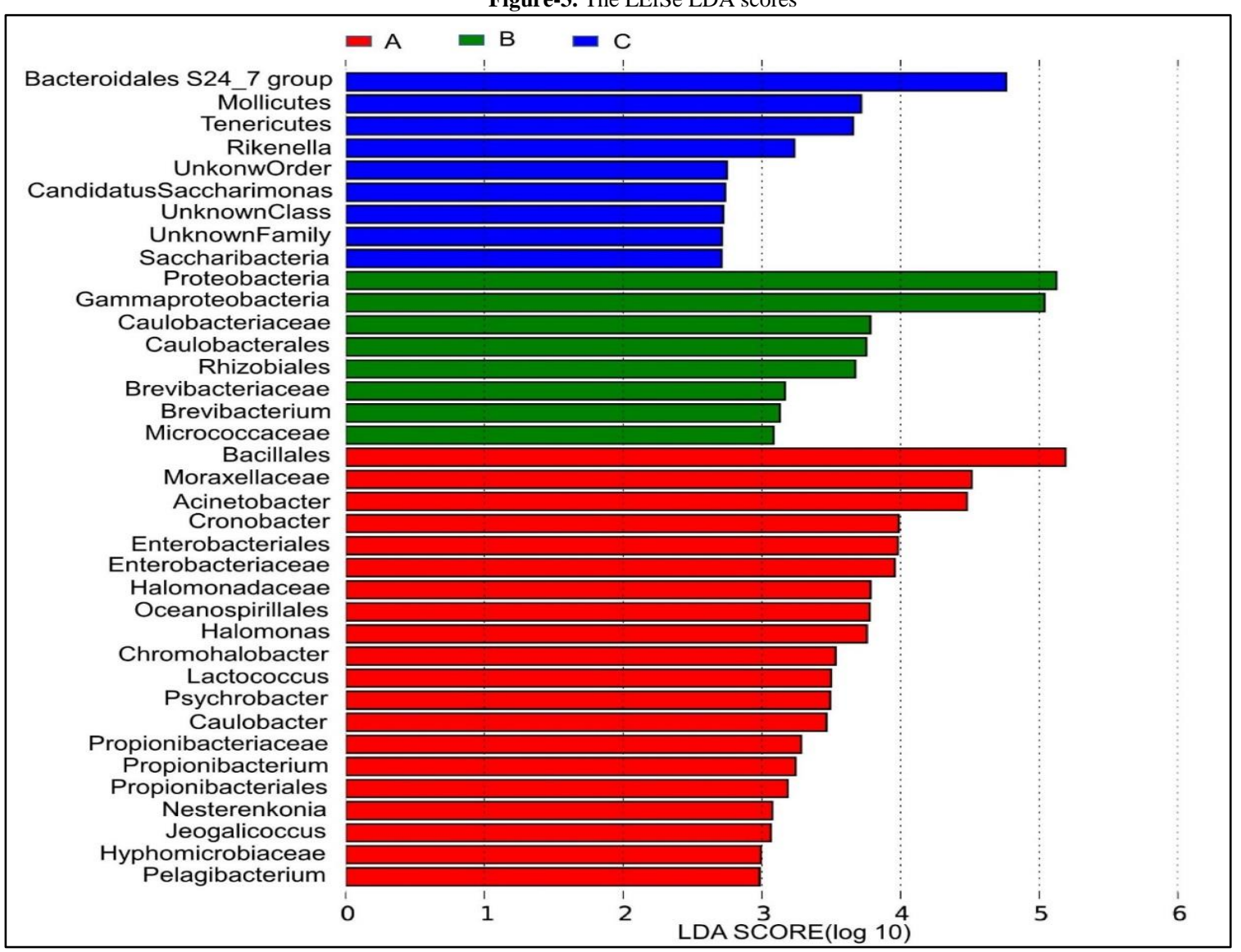




\subsection{Function Prediction}

The functions of the lung microbiota of healthy rats were examined by metagenomic prediction using PICRUSt. The metagenomics prediction showed the most abundant functional classes of lung microbiota were transporters, ATP-binding cassette (ABC) transporters, DNA repair and recombination proteins, ribosomes, two-component systems, purine metabolism, transcription factors, peptidases and pyrimidine metabolism (Figure-4).

Figure-4. The COG Function Prediction

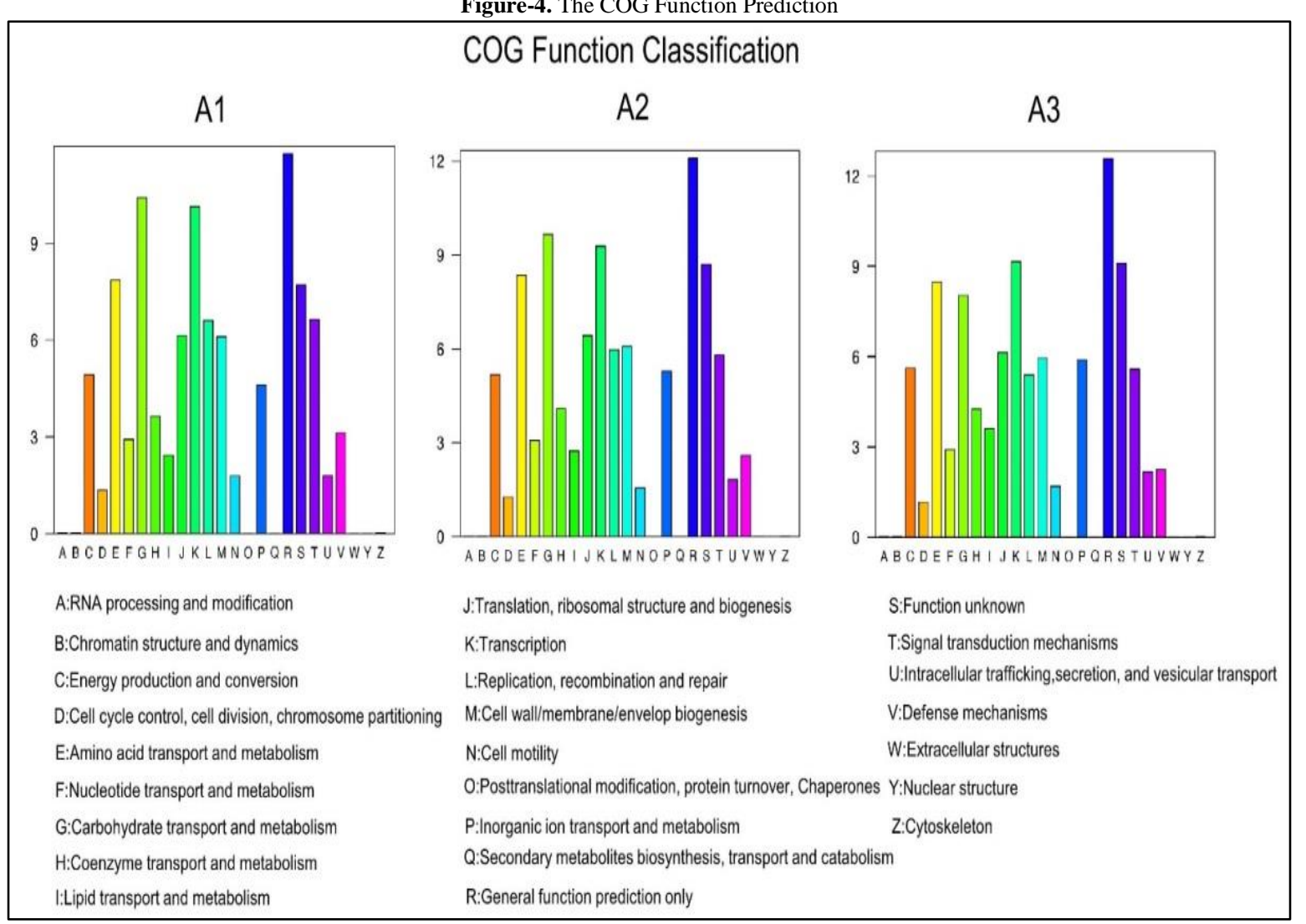

\section{Conclusion}

In this study, the sampling and sequencing methods can accurately detect the colonized bacteria and avoid interference with non-colonized bacteria [12]. This study has found there is colonization of dominating bacteria in healthy rat's lungs, trachea and bronchia. There was no significant difference in the abundance and diversity between the intestinal microbiota and the lung microbiota. The structure analysis revealed a large number of normal bacteria between the lungs and the intestine. This suggests the lungs, trachea and bronchia of healthy rats shared some persistent colonization of bacteria with the intestine. Moreover, we also found higher diversity within the lung microbiota when compared to the intestinal microbiota. There were differences in the types of dominant bacteria in the trachea, bronchus, lung and intestinal samples when microbiota structure was analyzed. These results indicate that bacteria colonization is associated with anatomical location of the sample as well as the physiological status of the individual. Compared with the sheep and canine model, there were significant differences in the species diversity and abundance of the lower respiratory tract microbiota in the rat model. As Proteobacteria in canine and Bacillus in sheep, Firmicutes is the dominant bacteria in rats (Aaron et al.2016; Laura et al. 2016). The abundance of actinomycetes in rat lung samples was considerably lower than that reported for sheep and canines. These differences may be associated with differences in species, habits, diet, feeding conditions, and sampling methods.

The functional capacity of lung microbiota was predicted by PICRUSt. The most abundant KOs were KOs related to transports and $\mathrm{ABC}$ transports, which were reported as the largest known protein families and widespread in bacteria. It demonstrated that the lung microbiota could sense the environmental stimuli and might involve in the development of lung-related diseases.

It is noteworthy that Lactococcus lactis, a human pathogen, was detected in rat lungs but not in the intestine. Acinetobacter lillmania (relative abundance $17.86 \%$ ) and Acinetobacter lwoffii (relative abundance $6.53 \%$ ) were identified in sample A3. The Acinetobacter lwoffii as a human pathogen in clinics and can cause lower respiratory tract infection, intracranial infection, sepsis, and more. The detection of Acinetobacter lwoffii suggest there are human pathogens colonized in the lower respiratory tract of rats.

In conclusion, our research suggests that the trachea, bronchia and lungs of healthy rats are colonized by rich microbial populations that differ from the gut microbiota. Further studies are needed to explore the specific mechanism and metabolic pathways of lung microbiota in healthy and diseased airway. 


\section{Funding}

This project was supported by Scientific Research and Innovative Experimental Program of Chongqing Medical University (No. 201509)

\section{References}

[1] Brzozowski, T. and Konturek, S. J., 2011. "Stress and the gut: pathophysiology, clinical conse quences, diagnostic approach and treatment options." J. Physiol Pharmacol, vol. 62, pp. 591-599.

[2] Keku, T. O., Dulal, S., and Deveaux, A., 2015. "The gastrointestinal microbiota and colorectal cancer." Am. J. Physiol Gastrointest Liver Physiol, vol. 308, p. G351-G363.

[3] Turnbaugh, P. J., Ley, R. E., and Mahowald, M. A., 2006. "An obesity-associated gut microbiome with increased capacity for energy harvest." Nature, vol. 444, pp. 10237-1031.

[4] Karlsson, F., Tremarokli, V., and Nielsen, J., 2013. "Assessing the human gut microbiota in met abolic diseases." Diabetes, vol. 62, pp. 3341-3349.

[5] Hilty, M., Burke, C., and Pedro, H., 2010. "Disordered microbial communities in asthmatic air ways." PLoS One, vol. 5, p. e8578.

[6] Gollwitzer, E. S. and Marsland, B. J., 2014. "Microbiota abnormalities in inflammatory airway dis easespotential for therapy." Pharmacol Ther, vol. 141, pp. 32-39.

[7] Hampton, T. H., Green, D. M., and Cutting, G. R., 2014. "The microbiome in pediatric cystic fibrosis patients: the role of shared environment suggests a window of intervention." Microbiom, vol. 2, p. 14.

[8] Hosgood, H. r., Sapkota, A. R., and Rothman, N., 2014. "The potential role of lung mi crobiota in lung cancer attributed to household coal burning exposures." Environ Mol Mutagen, vol. 55, pp. 643-651.

[9] Doud, M., Zeng, E., and Schneper, L., 2009. "Approaches to analyse dynamic microbial commu nities such as those seen in cystic fibrosis lung." Hum Genomics, vol. 3, pp. 246-256.

[10] Aaron, C. E., Alexa, R. P., and Megan, E. G., 2016. "Composition and predicted metabolic capac ity of upper and lower airway microbiota of healthy dogs in relation to the fecal microbiota." Plos One, Available: https://journals.plos.org/plosone/article?id=10.1371/journal.pone.0154646

[11] Laura, G., Steven, W., and Jolinda, P., 2016. "Variability of the sheep lung microbiota." Ap plied and Environmental Microbiology, vol. 82, pp. 3225-3238.

[12] Zhiying, T., Zhibang, Y., Jiye, G., Lili, Z., Renju, J., and Ying, J., 2015. "Lower esophageal microbiota species are affected by the eradication of Helicobacter pylori infection using antibiotics." Experimental And Therapeutic Medicineexperimental Medicine, vol. 9, pp. 685-692. 